\title{
A Feature Level Fusion in Similarity Matching to Content-Based Image Retrieval
}

\author{
Md. Mahmudur Rahman, Bipin C. Desai \\ Computer Science Department \\ Concordia University \\ Montreal, QC, CANADA \\ mah_rahm@cs.concordia.ca
}

\author{
Prabir Bhattacharya \\ CIISE \\ Concordia University \\ Montreal, QC, CANADA \\ prabir@ece.concordia.ca
}

\begin{abstract}
This paper presents a fusion-based similarity matching framework for content-based image retrieval on a combination of global, semi-global and local region specific features at different levels of abstraction. In this framework, an image is represented by global color and edge histogram descriptors, semi-global color and texture descriptors from grid based overlapping sub-images and local color features from a clustering-based segmented regions. As a result, image similarities are obtained through a weighted combination of overall similarity fusing global, semi-global and local region-based image level similarities. This fusing approach decreases the impact of inaccurate segmentation and increases retrieval effectiveness as constituent features are of a complementary nature. The experimental results on a general-purpose image database indicate that the aggregation or fusion-based technique provides an effective and flexible tool for similarity calculation based on a combination of descriptors from different levels of image representation.
\end{abstract}

Keywords: Image retrieval, feature extraction, clustering, segmentation, similarity measure.

\section{Introduction}

The exponential growth of image data has created a compelling need for innovative tools for managing, retrieving, and visualizing images from large collection. Many applications such as digital libraries, image search engines, medical decision support systems require effective and efficient image retrieval techniques to access the images based on their contents, commonly known as content-based image retrieval (CBIR) $[1,2]$. CBIR systems computes relevance of query and database images based on the visual similarity of low-level (e.g., color, texture, shape, edge etc.) image features $[1,2,3,4,5,6]$. Over the last few years, research interest into CBIR has grown rapidly from database, computer vision, pattern recognition, machine learning, and information retrieval communities. While much research effort has been made on the development of the CBIR systems and various methods have been proposed $[1,2]$, the performance is still limited. The limited retrieval accuracy is mainly because of the miss- match between user semantic concepts and system generated low-level image features [1]. In general, the similarity comparison is performed either globally from the entire image or locally from automatically derived segmented regions. However, global features lack spatial feature distribution information and fail to capture enough semantic information due to limited descriptive power. To retain spatial information, some fixed grid based image partitioning techniques have been proposed with moderate success toward semantic-based retrieval [2]. Recently, region-based image retrieval (RBIR) have been studied widely $[3,4,5,6]$, where each image is first segmented automatically into variable number of homogeneous regions, features of each regions are extracted and images are retrieved based on the similarity between regions. Although, there is a strong correlation between segmented regions and real-world objects, accurate segmentation of generalpurpose images is still an unsolved problem [5]. In summary, current CBIR systems can be distinguished as global, semi-global or region specific in nature based on their feature representation and employed similarity matching functions. However, it is assumed that more perceptually meaningful results might be obtained if similarity matching functions from different representations at different levels of abstraction are fused or aggregated in a combined fashion as features from different levels can complement each other.

Based on this assumption, we propose an image retrieval technique with a novel fusion-based similarity matching function, where the objective is to match the entire images, including backgrounds and main objects. In addition, we also propose a region-based similarity matching technique, where the images are first segmented into a set of homogeneous regions by a modified k-means clustering algorithm. Next, the image level similarity is defined through a region to region similarity, and then combining the results at image level. The proposed approaches are presented in the next sections, where Section 2, 3, and 4 present global, semi-global and region level feature extraction and distance measure functions and Section 5 presents the proposed fusion-based similarity matching function. Experimental results are provided in Section 6 and finally, we conclude in Section 7. 


\section{Global features and distance measure}

The performance of a CBIR system mainly depends on the particular image representation and similarity matching function employed. We have extracted color and edge related features for our image representation at global level. Color and its histogram based representation is the most useful and widely used low-level feature in CBIR [1]. For color feature, a 108-dimensional color histogram is created in vector form on HSV (Hue, Saturation, Value) color space. In HSV space, the colors correlates well and can be matched in a way that is consistent with human perception. HSV space is uniformly quantized into 12 bins for hue(each bin consisting of a range of $30^{\circ}$ ), 3 bins for saturation and 3 bins for value, which results in 108 bins for color histogram. To represent the shape feature on a global level, a histogram of edge direction is constructed. The edge information contained in the images is processed and generated by using the Canny edge detection (with $\sigma=1$, Gaussian masks of size $=9$, low threshold $=$ 1 , and high threshold $=255$ ) algorithm [7].The corresponding edge directions are quantized into 72 bins of $5^{\circ}$ each. Scale invariance is achieved by normalizing this histograms with respect to the number of edge points in the image.

Since, the combined dimension of the color histogram and texture feature vector (108 for color and 72 for edge for a total of 180) is large, we need to apply some dimension reduction technique to reduce the computational complexity and the logical database size. The problem of selecting most representative feature attributes, commonly known as dimension reduction, has been examined by principal component analysis (PCA) in many pattern recognition areas [8]. The basic idea of $P C A$ is to find $m$ linearly transformed components so that they explain the maximum amount of variances in the input data. The mathematical steps used to describe this method is as follows:

given a set of $N$ feature vectors (training samples) $\mathbf{x}_{\mathbf{i}} \in \Re^{d} \mid i=(1 \cdots N)$, the mean vector $(\mu)$ and covariance matrix $(C)$ is estimated from these samples by applying maximum likelihood principle. Let $\nu_{k}$ and $\lambda_{k}$ be the eigenvectors and the eigenvalues of $C$, then they satisfy the following:

$$
\lambda_{k}=\sum_{i=1}^{N}\left(\nu_{k}^{T}\left(\mathbf{x}_{i}-\mu\right)\right)^{2}
$$

Here, $\sum_{k=1}^{d} \lambda_{k}$ accounts for the total variance of the original feature vectors set. Now, the $P C A$ method tries to approximate the original feature space using an $m$-dimensional feature vector, that is using $m$ largest eigenvalues account for a large percentage of variance, where typically $m<<\min (d, N)$. These $m$ eigenvectors span a subspace, where $\mathbf{V}=\left[\mathbf{v}_{1}, \mathbf{v}_{2}, \cdots, \mathbf{v}_{m}\right]$ is the $d \times m$-dimensional matrix that contains orthogonal basis vectors of the feature space in its columns. The $m \times d$ transformation $\mathbf{V}^{T}$ transforms the original

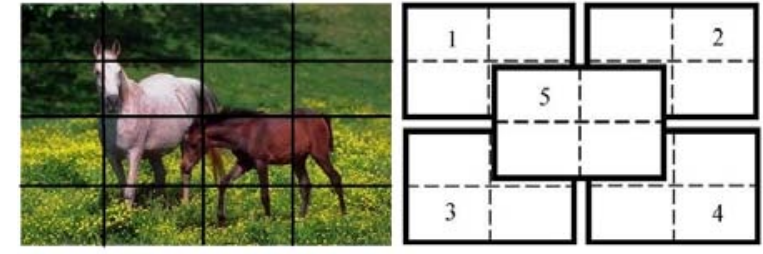

Figure 1: Region generation from sub-images

feature vector from $\Re^{d} \rightarrow \Re^{m}$ ones. That is

$$
\mathbf{V}^{T}\left(\mathbf{x}_{i}-\mu\right)=\mathbf{y}_{i}, i=1 \cdots N
$$

where $\mathbf{y}_{i} \in \Re^{m}$ and $k$ th component of the $\mathbf{y}_{i}$ vector is called the $k$ th principal component (PC) of the original feature vector $\mathbf{x}_{i}$. So, the feature vector in the original $\Re^{d}$ space of images can be projected on to the $\Re^{m}$ space via the transformation of $\mathbf{V}^{T}[8]$.

In pattern recognition applications, $P C A$ has been mainly employed to reduce the dimension of a single feature vector or a composite feature vector combined with various features. In this work, we consider the later approach [11], where color histogram and edgebased features vectors are combined or concatenated to form a single vector as $f^{\text {global }}=\left(f_{\mathrm{c}}+f_{\mathrm{e}}\right) \in \Re^{d}$, where $f_{\mathrm{c}}$ and $f_{\mathrm{e}}$ are color and edge descriptors respectively. This composite feature vector is converted to a lowdimensional feature vector $\hat{f}^{\text {global }} \in \Re^{m}$ in PCA subspace. Now, for comparing query image $Q$ and target image $T$ in the database, Euclidean distance measure is used as

$$
\operatorname{DIS}_{\mathrm{g}}(Q, T)=\left\|\hat{f}_{\mathrm{Q}}^{\text {global }}-\hat{f}_{\mathrm{T}}^{\text {global }}\right\|=\sqrt{\sum_{i=1}^{m}\left(\hat{f}_{\mathrm{Q}_{i}}-\hat{f}_{\mathrm{T}_{i}}\right)}
$$

However, the main drawback of the global feature representation is that spatial information about object is discarded and is failed to narrow the semantic gap due to limited description power.

\section{Semi-global features and dis- tance measure}

Semi-global feature representation attempts to overcome the drawback of global features [2]. For semiglobal features, a grid based approach is taken into account to divide the images in five overlapping subimages based on the assumption that main object generally located at the center of the image. These subimages are obtained by first dividing the entire image space into 16 non overlapping sub-images. From there, we cluster four connected sub-images to generate five different clusters of overlapping sub-images as shown in Fig. 1. The first (mean), second (standard deviation) and third (skewness) central moments of each color channel are extracted for the semi-global color feature vector in HSV color space [9]. Texture features are extracted from the grey level co-occurrence matrix (GLCM) [10]. GLCM is defined as a sample of the 
joint probability density of the gray levels of two pixels separated by a given displacement. Second order moments, such as energy, maximum probability, entropy, contrast and inverse difference moment were measured based on the GLCM. Color and texture feature vectors are normalized and combined to form a joint feature vector of 14-dimensions (9 for color and 5 for texture).

Let, $Q^{r}$ and $T^{r}$ represent the $r$ th region in the query image $Q$ and database image $T$. Then $D_{\mathrm{c}}(Q, T)=$ $\sum_{r=1}^{5} D_{\mathrm{c}}\left(Q^{r}, T^{r}\right)$ and $D_{\mathrm{t}}(Q, T)=\sum_{r=1}^{5} D_{\mathrm{t}}\left(Q^{r}, T^{r}\right)$ describe the image level color and texture distance measures, where $D_{\mathrm{c}}\left(Q^{r}, T^{r}\right)$ and $D_{\mathrm{t}}\left(Q^{r}, T^{r}\right)$ represents the distance measures of color and texture feature of each region based on Euclidean distance respectively. Finally, we obtain the overall semi-global image level similarity as

$$
\operatorname{DIS}_{\mathrm{sg}}(Q, T)=\omega_{\mathrm{c}} D_{\mathrm{c}}(Q, T)+\omega_{\mathrm{t}} D_{\mathrm{t}}(Q, T),
$$

where $\omega_{\mathrm{c}}$ and $\omega_{\mathrm{t}}$ are weights for each feature distance measure subject to $\omega_{\mathrm{c}}+\omega_{\mathrm{t}}=1$ and adjusted as $\omega_{\mathrm{c}}=0.7$ and $\omega_{\mathrm{t}}=0.3$ in the experiment. However, an obvious drawback of this approach is that, it is sensitive to shifting, scaling, and rotation because image are represented by a set of local properties and fixed partitioning scheme might not match with the actual semantic partitioning of objects.

\section{Local region specific features and distance measure}

Region-based image retrieval (RBIR) aims to overcome the limitations of global and semi-global retrieval approaches by fragmenting an image automatically into a set of homogeneous regions based on color and/or texture properties. Then each region can be described by means of local features. The overall similarity between two images is calculated based on all the corresponding region-based local features [5, 4]. In this section, we propose a clustering-based image segmentation technique and an image to image level similarity matching function based on clusters or regions generated from the segmentation.

\subsection{Clustering-based region generation}

A fast k-means color-clustering is utilized to segment the images since color is the most important feature for general-purpose images [12]. The clustering method is fully automatic and unsupervised in nature that can adaptively updates the number of regions as an iterative process as a fact that the number of region is unknown before the segmentation. To segment an image into regions, the image is first partitioned into nonoverlapping 2 by 2 square image-blocks. The average color components of each image-block are extracted as feature vector in HSV color space. The k-means algorithm is used to cluster the feature vectors into several groups with each group corresponding to one spatial region in the segmented image.
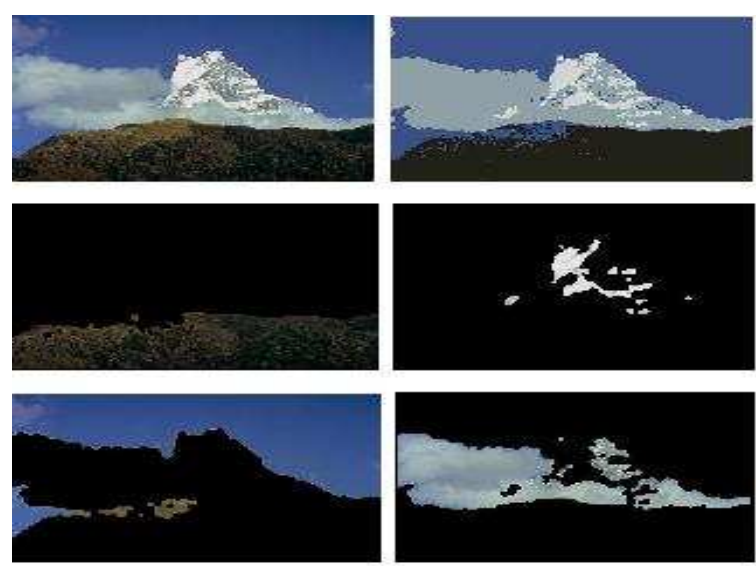

Figure 2: Region detection from segmentation

Suppose there is a set $X=\left\{x_{i}, \cdots, x_{L}\right\}$ of $L$ blocks for each image to be segmented. The goal of the $\mathrm{k}$ means algorithm is to partition the blocks into $k$ clusters with means $V=\left\{\mu_{i}, \cdots, \mu_{k}\right\}$ such that

$$
D(k)=\sum_{i=1}^{k} \sum_{x_{j} \in C_{i}}\left(x_{j}-\mu_{i}\right)^{2}
$$

is minimized, where $\mu_{i}$ is the centroid or mean of the $i$-th cluster $C_{i}$. We primarily select the number of clusters with $k=2$ and gradually increase $k$ until a stop constraint is satisfied. Two stop constraints are as follows: if $D(i)$ (total number of clusters $k=i$ at the $i$-th iteration) is less than a threshold or $D(i)-D(i-1)$, is below another threshold, which are found out empirically from our test database. Since clustering is performed in the feature space, blocks in each cluster do not necessarily form a connected region in the image space. To remove small isolated regions or holes and smooth region boundaries in each binary masked segmented regions, morphological opening and closing operations are applied as a postprocessing filtering approach. Fig. 2 shows the segmentation results of a sample database image with four regions after applying morphological close-open filtering operation. The non-connected property of some regions preserves the natural clustering of an object that is good enough for retrieval. To represent each region with local features, we consider information on weight and color-texture as in Ref. [4].

Let, $w_{T_{R_{i}}}$ is the weight of region $R_{i}$ of image $T$. We define $w_{T_{R_{i}}}=\frac{N_{T_{R_{i}}}}{N_{T}}$, where $N_{T_{R_{i}}}$ is the number of blocks in region $R_{i}$ and $N_{T}$ is the total number of blocks in image $T$. Color feature $f_{R_{i}}^{c}$ of each region $R_{i}$ is a $3-\mathrm{D}$ vector and represented by the k-means cluster center, i.e., the average value for each of the three color channels in HSV space of all the image blocks in this region. Texture feature of each region is measured in an indirect way by considering the cross-correlation among color channels due to the off diagonal of the $3 \times 3$ covariance matrix $C_{T_{R_{i}}}$ of region $R_{i}$ in image $T$ 
and is estimated as

$$
C_{T_{R_{i}}}=\frac{1}{N_{T_{R_{i}}}-1} \sum_{j=1}^{N_{T_{R_{i}}}}\left(f_{x_{j}}^{c}-f_{R_{i}}^{c}\right)\left(f_{x_{j}}^{c}-f_{R_{i}}^{c}\right)^{T}
$$

where $f_{x_{j}}^{c}$ is the color vector of block $x_{j}$ and $f_{R_{i}}^{c}$ is the feature vector of the region $R_{i}$ or the cluster centroid.

\subsection{Image level region-based similarity measure}

To compute the distance between two regions $R_{i}$ and $R_{j}$ of query image $Q$ and database image $T$ respectively, we apply Bhattacharyya distance metric [12] as follows:

$$
\begin{gathered}
D\left(R_{i}, R_{j}\right)=\frac{1}{8}\left(f_{R_{i}}^{c}-f_{R_{j}}^{c}\right)^{T}\left[\frac{\left(C_{Q_{R_{i}}}+C_{T_{R_{j}}}\right)}{2}\right]^{-1} \\
\left(f_{R_{i}}^{c}-f_{R_{j}}^{c}\right)+\frac{1}{2} \ln \frac{\left|\frac{\left(C_{Q_{R_{i}}}+C_{T_{R_{j}}}\right)}{2}\right|}{\sqrt{\left|C_{Q_{R_{i}}}\right|\left|C_{T_{R_{j}}}\right|}}
\end{gathered}
$$

where $f_{R_{i}}^{c}$ and $f_{R_{j}}^{c}$ are the region feature vectors, and $C_{Q_{R_{i}}}$ and $C_{T_{R_{i}}}$ are the covariance matrices of region $R_{i}$ and $R_{j}$ of query image $Q$ and target image $T$ respectively. Equation (7) is composed of two terms, the first one being the distance between feature vectors of image regions, while the second term gives the class separability due to the difference between covariance matrices.

The distance between image $Q$ and $T$ is measured by integrating properties of all the regions in the images, which is robust against poor segmentation. We allow one region of an image to be matched to several regions of another image. Suppose, there are $M$ regions in image $Q$ and $N$ regions in image $T$. The image-level distance between two images $Q$ and $T$ is

$$
\operatorname{DIS}_{1}(Q, T)=\frac{\sum_{i=1}^{M} w_{Q_{R_{i}}} R_{i}(T)+\sum_{j=1}^{N} w_{T_{R_{j}}} R_{j}(Q)}{2}
$$

where $w_{Q_{R_{i}}}$ and $w_{T_{R_{j}}}$ are the weights for region $i$ of image $Q$ and region $j$ of image $T$ respectively. For each region $i \in M$ in image $Q, R_{i}(T)$ is defined as the minimum distance between this region and any region $j \in N$ in image $T$ :

$$
R_{i}(T)=\min \left(D\left(R_{i}, R_{1}\right), \cdots, D\left(R_{i}, R_{j}\right)\right)
$$

Similarly,

$$
R_{j}(Q)=\min \left(D\left(R_{j}, R_{1}\right), \cdots, D\left(R_{j}, R_{i}\right)\right)
$$

This definition of image-level distance between two images captured by the overall distance between the region sets of two images is a balanced scheme in distance measure between regional and global matching.

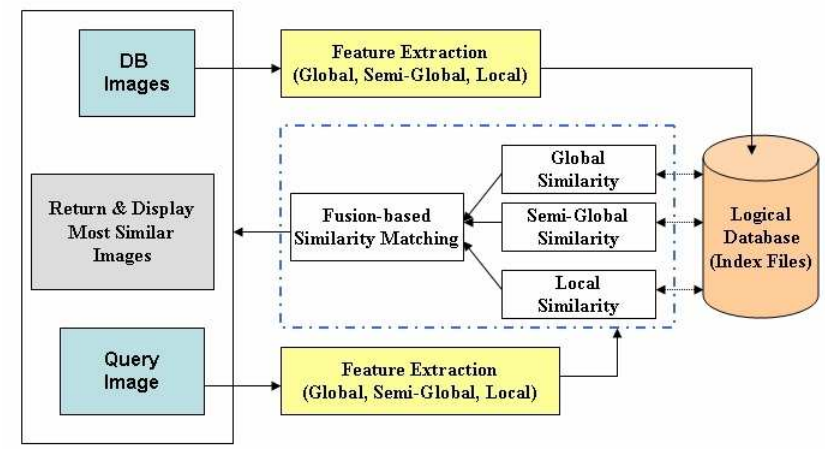

Figure 3: Block diagram of the proposed image retrieval technique

\section{Fusion-based Overall Image level similarity}

The overall image-level similarity is measured by fusing of a weighted combination of individual similarity measures. In the previous sections, the global, semi-global and local distance measures as $\operatorname{DIS}_{\mathrm{g}}(Q, T)$, $\operatorname{DIS}_{\mathrm{sg}}(Q, T)$ and $\operatorname{DIS}_{1}(Q, T)$ are defined. Once the distance functions are measured, it can be easily normalized and converted to similarity measures. Similarity measure is a quantity that reflects the strength of relationship between two features and is usually normalized into a range of $[0,1][1]$. Generally, a similarity measure function is the converse of a distance function. So, when similarity is one (i.e. exactly similar), the distance is zero and when the similarity is zero (i.e. very different), the distance is one. For all the above distances, the following function is used to transform the distance measures into a similarity measures as $S(Q, T)=\exp ^{-\operatorname{DIS}(Q, T) / \sigma_{\operatorname{DIS}(Q, T)}}$, where $\sigma_{\operatorname{DIS}(Q, T)}^{2}$ is the distance variance computed for each distance measure separately over a sample image set. After the similarity measures of each representation are determined as $\mathrm{S}_{\mathrm{g}}(Q, T), \mathrm{S}_{\mathrm{sg}}(Q, T)$ and $\mathrm{S}_{\mathrm{l}}(Q, T)$, we can aggregate or fuse them into a single similarity matching function as follows:

$$
\mathrm{S}(Q, T)=w_{\mathrm{g}} \mathrm{S}_{\mathrm{g}}(Q, T)+w_{\mathrm{sg}} \mathrm{S}_{\mathrm{sg}}(Q, T)+w_{\mathrm{l}} \mathrm{S}_{\mathrm{l}}(Q, T)
$$

Here, $w_{\mathrm{g}}, w_{\mathrm{sg}}$, and $w_{\mathrm{l}}$ are non-negative weighting factors of different feature level similarities with normalization $\left(w_{\mathrm{g}}+w_{\mathrm{sg}}+w_{\mathrm{l}}=1\right)$, which needs to be selected experimentally.

Figure 3 shows the block diagram of the proposed fusion-based similarity matching technique with feature descriptors at different levels for image retrieval.

\section{Experimental Results}

To evaluate the effectiveness of the proposed framework, experiments were performed on a generalpurpose image database with 3000 images collected from COREL and IAPR image collection [13]. This database contains diverse images of 15 manually assigned semantical categories (Mountain, Beach, Archi- 


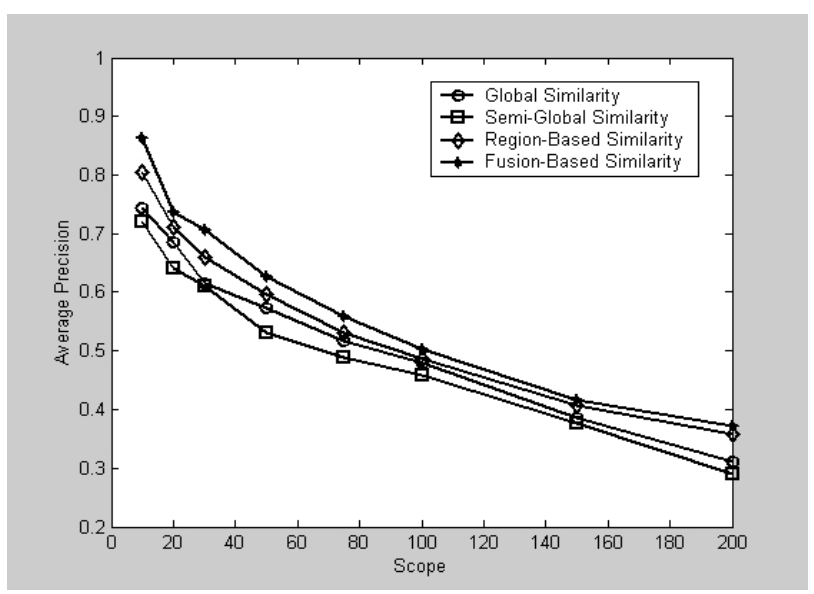

Figure 4: Average precision curves

tecture, Flower, Food, Animal etc.) with 200 images in each category. We have randomly selected 10 images from each categories (i.e., 150 images in total) as query images and used query-by-example method, where the query is specified by providing an example image to the system.

For a quantitative evaluation, the performances of individual and fusion-based similarity measures are compared based on average precision curves by evaluating top 200 returned results. Precision is the ratio of the number of relevant images returned to the total number of images returned. So, when the top $N$ (scope) images are considered and there are $R$ relevant images, the precision within top $N$ images is defined to be Precision $(N)=Q / R$. A high precision value means that there are few false alarms (i.e., the $\%$ of irrelevant images in the retrieval). A retrieved image is considered a correct match if it is in the same category as the query image. The performances of the similarity measures are compared based on average precision curves by evaluating top $N=\{10,20,50,70,100,150,200\}$ returned results.

We have assigned highest weight to the region based similarity measure as $w_{1}=0.6$, since it captures more details and close to semantic perceived by users. Global and semi-global similarity measures have more or less same performances in our experimental database, hence equal weights are assigned to both of them as $w_{\mathrm{g}}=0.2$ and $w_{\mathrm{sg}}=0.2$. Fig. 4, presents average precision curves, which clearly shows that fusion-based similarity measures outperformed the other three similarity measures, whereas semi-global similarity measure performed poorly among all of these. The result is expected as fusion-based similarity function decrease the impact of inaccurate segmentation and complement each other representation.

For qualitative evaluation purpose, Figure 5, 6, 7 and 8 show the snapshots of the retrieval interface (returns top most 15 similar images) for a query image of architecture category based on global, semi-global, region and fusion similarity matching functions. From Figure 8 and 6 , it is clear that there is only one missmatch (second image in the third row under mountain category) for fusion-based similarity matching func-

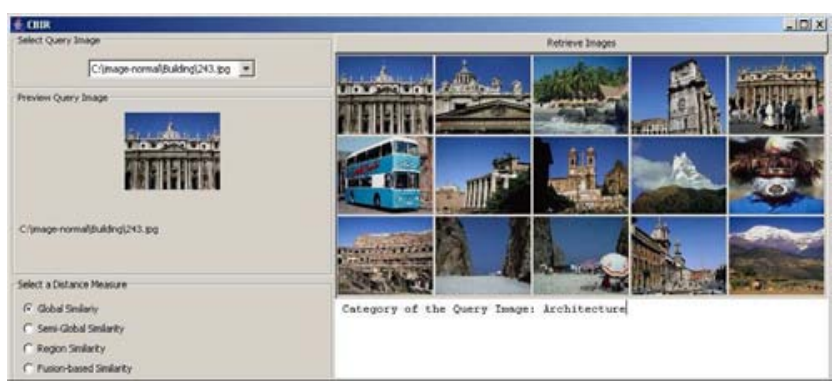

Figure 5: Snapshot of retrieval interface based on global similarity

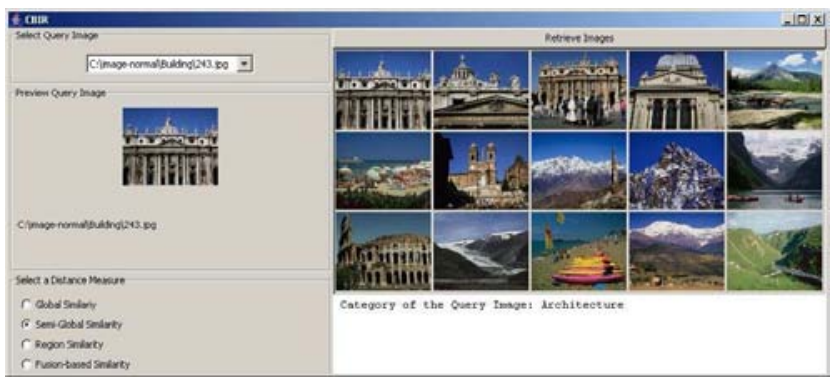

Figure 6: Snapshot of retrieval interface based on semiglobal similarity

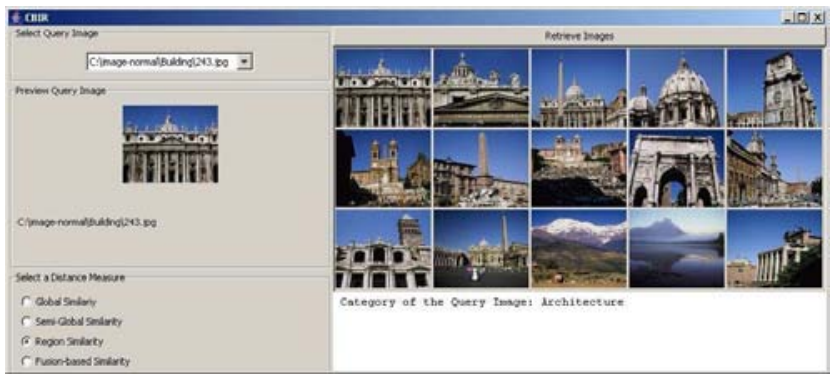

Figure 7: Snapshot of retrieval interface based on region similarity

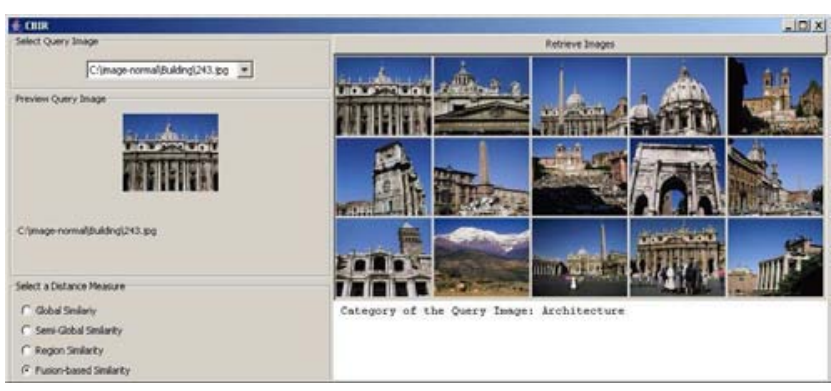

Figure 8: Snapshot of retrieval interface based on fusion similarity 
tion and nine miss-matches for semi-global similarity matching function for this particular query image. Whereas, Figure 5 and 7 show that the performances of global and region based similarities are better then the semi-global one. However, the performance of the fusion-based similarity matching function is the best among these measures in this case which comply with our assumption and the average precision curves as shown in Figure 4.

\section{Conclusions}

Similarity matching is one of the major functions in a CBIR system and calculating it effectively is one of the key challenges. In this paper, a region based image level similarity matching function is proposed based on automatically derived segmented regions and a fusion-based similarity matching function is proposed based on a weighted combination of similarity measures at different level of representation. We observe that the segmentation-related uncertainties always exist due to inaccurate image segmentation especially for the images with indistinctive and irrelevant scenes. The global and semi-global features are utilized in combination with region specific features to decrease the impact of inaccurate segmentation and consequently performance degradation. We tested and evaluated the performances in a general-purpose image database with known ground truth, which showed promising results.

\section{References}

[1] A. Smeulder, M. Worring, S. Santini, A. Gupta, R. Jain, Content-Based Image Retrieval at the End of the Early Years., IEEE Trans. on Pattern Anal. and Machine Intell., 22:1349-1380, 2000.

[2] Y. Rui, T. S. Huang, and S.-F. Chang, Image Retrieval: Current Techniques, Promising Directions and Open Issues. Journal of Visual Communication and Image Representation, 10(4): 39-62, 1999.

[3] C. Carson, S. Belongie, H. Greenspan, and J. Malik, Blobworld: Image Segmentation Using Expectation-Maximization and Its Application to Image Querying, IEEE Trans. on Pattern Analysis and Machine Intelligence, 24(8): 1026-1038, 2002.

[4] S. Ardizzoni, I. Bartolini, and M. Patella, Windsurf: Region-based image retrieval using wavelets, in DEXA Workshop, 167-173, 1999.

[5] J. Z. Wang, J. Li, and G. Wiederhold, SIMPLIcity: Semantics-Sensitive Integrated Matching for Picture LIbraries, IEEE Trans. on Pattern Analysis and Machine Intelligence, 23(9): 947963, 2001.
[6] W. Y. Ma, B. Manjunath. NaTra: A toolbox for navigating large image databases. Proc. IEEE Int. Conf. Image Processing, pp. 568-571, Santa Barbara, 1997.

[7] J. Canny, A computational approach to edge detection., IEEE Trans. Pattern Anal. Machine Intell., 8: 679-698, 1986.

[8] A. K. Jain, B. Bhandrasekaran, Dimensionality and sample size considerations in pattern recognition practice., Handbook of Statistics, 2: 835-855, 1987.

[9] M. Stricker, M. Orengo, Similarity of color images: Storage and Retrieval for Image and Video Databases., SPIE, vol. 2420, 1995.

[10] S. Aksoy, R. M. Haralick, Texture Analysis in Machine Vision., Chapter Using Texture in Image Similarity and Retrieval, Series on Machine Perception and Artificial Intelligence., World Scientific, 2000.

[11] M. M. Rahman , P. Bhattacharya and B.C. Desai, Similarity Searching in Image Retrieval with Statistical Distance Measure and Supervised Learning, International Conference on Advances in Pattern Recognition (ICAPR), LNCS, 3686 : 315-324, 2005.

[12] K. Fukunaga, Introduction to Statistical Pattern Recognition, Second ed., Academic Press, 1990.

[13] C. Leung, H. Ip, Benchmarking for Content Based Visual Information Search, Fourth International Conference on Visual Information Systems (VISUAL2000), LNCS, 1929:442-456, 2000. 